\title{
Evaluation of Endoscopic Characteristics of Upper Gastrointestinal Polyps in Patients with Familial Adenomatous Polyposis
}

\author{
Seyed Reza Fatemi,Azadeh Safaee,Sara Pasha, Mohamad Amin Pourhoseingholi*, \\ Rasool Bahrainei, Mahsa Molaei
}

\begin{abstract}
Background: Familial adenomatous polyposis (FAP) is a disease inherited in an autosomal dominant fashion. Most FAP patients develop upper gastrointestinal polyps; especially those in the antrum and duodenum are usually neoplastic. The aim of this study was to evaluate the prevalence of gastroduodenal polyps in Iranian FAP patients. Materials and Methods: 28 patients affected by FAP underwent front-view and side-view endoscopy. Papillary biopsies were performed in all patients. Location of polyps, their number and size, pathology study, patient general information (gender, age, family history of FAP or colorectal cancer and gastroduodenal polyps) were analyzed. Results: Gastric polyps were seen in $39.3 \%$ of patients. Some $72.7 \%$ of the affected individuals had fundic gland polyps and $36.36 \%$ had hyperplastic polyps. Duodenal adenoma was observed in $25 \%$ of patients. While $57 \%$ of patients had tubular adenoma with low grade dysplasia, $42.8 \%$ showed tubulovillous adenoma with low grade dysplasia. Conclusions: Findings of this study indicated that the prevalence of gastroduodenal polyps in FAP patients is high and dysplasia may be evident in duodenal polyps. Therefore, it appears that routine gastroduodenal endoscopy in FAP patients is necessary.
\end{abstract}

Keywords: Gastric polyp - duodenal adenoma - familial adenomatous polyposis - spigelman stage

Asian Pac J Cancer Prev, 15 (16), 6945-6948

\section{Introduction}

Colorectal cancer (CRC) is one of the most common cancers worldwide and its burden is increasing in Asian Pacific region countries including Iran (Pourhoseingholi et al., 2009; Somi et al., 2014). CRC exists in a more common sporadic form and less common hereditary form, such as the Lynch syndrome, familial adenomatous polyposis (FAP) and other rare syndromes (Nizam et al., 2013). FAP is a disease inherited in an autosomal dominant fashion, which due to a mutation on chromosome five, leads to the development of up to several thousands of colorectal adenomas and a number of extra colonic manifestation (Poovorawan et al., 2012). Extra-colonic manifestations (such as desmoids tumors and duodenal adenomatousis) are the important reasons of death in polyposis population. All untreated patients will develop colorectal cancer (Samalavicius., 2008).

Upper gastrointestinal malignancy is the major cause of death after colectomy. The most FAP patients develop upper gastrointestinal polyps, especially those in the antrum and duodenum are usually neoplastic (Offerhaus et al., 1999). In one study $83 \%$ of FAP patients had gastroduodenal polyps, $60 \%$ gastric polyps and $70 \%$ duodenal polyps (Goedde et al., 1992). Fundic gland polyps are the commonest gastric lesion in FAP. In the general population these polyps are considered benign and no have malignant transformation potential. However, rarely, in FAP patients fundic gland polyps have been distinguished as precursor lesions from which invasive cancer may develop (Garrean et al., 2008). After fundic gland polyps duodenum is the commonest site of polyp development in FAP patients (Park et al., 2011). Duodenal polyps in FAP patients are classified as adenomatous polyps, and considered as precancerous lesions for duodenal adenocarcinoma (Spigelman et al., 1994). In one study normal-appearing papilla was adenomatous in 50 percent of cases (Church et al., 1992).

The spigelman classification, in which the number of adenomas, the size, architecture and degree of dysplasia account for the scoring system, provides a guide for follow up in FAP patients (Offerhaus et al., 1999). The spigelman classification in 366 classifiable patients was reported as: stage $0,123(34 \%)$, stage $1,55(15 \%)$, stage II, 97(27\%) stage III, $64(17 \%)$ and stage IV, 27(7\%) and two patients $(0.5 \%)$ had duodenal carcinoma. Also it is proved that the spigelman stage will progress with time and patient age (Bulow et al., 2004). Another study 
reported a high incidence rate of duodenal polyps in FAP patients $(79.3 \%)$ and all of patient had low spigelman score (Cordero-Fernandez., 2009).

Findings of another research show that polyps $\geq 10 \mathrm{~mm}$ in diameter or polyps in the second part of duodenum are independent risk factors for being malignant lesions (Jung et al., 2010).

Because there is not much information about upper gastrointestinal polyps in FAP patients in Iran, the present study was designed to investigate about endoscopic characteristics of upper gastrointestinal polyps in Iranian FAP patients.

\section{Materials and Methods}

This study was designed as a cross-sectional study. Cases were selected among patients who have been registered at gastroenterology and liver disease Research Center, Taleghani Hospital (since 2007-2012). 28 patients (from 23 families) participated in this study (the diagnosis of FAP was based on the presence of multiple adenomatous polyps in colon and rectum and APC gene study). Patients were interviewed to obtain general information, such as: gender, age, and age at diagnosis of FAP, family history of FAP or gastroduodenal polyps or cancer or colorectal cancer. Then the patients underwent upper GI endoscopy and the presence or absence of polyps, site, size and number of polyps, the appearance of polyps (sessile or pedunculated) and other abnormalities were observed by gastroenterologist by use of endoscopy.

Biopsy of polyps or polipectomy and biopsy of papilla even it appeared normal was taken. Side-view endoscopy in order to evaluate the duodenum and papilla region in more details was used. The specimens were sent to the laboratory for pathology study and the reports of pathology, based on number of sampling, site of polyps, histology and grade of dysplasia of polyps were obtained. The spigelman classification was used for the better clarification of duodenal involvement. Finally, all gathered information was analyzed and categorized by specified factors.

\section{Results}

A total of 28 patients (10 Females, 18 males) with the mean age of $37.4 \pm 14.52$ (range 19-69 y) entered to this study. Age distribution of patients was shown in Table 1.

2 patients $(7.1 \%)$ were affected by colorectal cancer (In association with FAP) at age of $25.42 .8 \%(n=12)$ and $28.5 \%(n=8)$ of patients were reported family history of colorectal cancer or FAP, respectively. Also, family history of Gastric polyp and duodenal polyp was observed in 7 patients $(25 \%)$ and 6 patients $(21.4 \%)$.

All of patients underwent front-view and side-view endoscopy (Table 2) and the findings

Were as the following: Gastric polyp was seen in 39.28 $\%$ of cases. (72.7\% of patients with gastric polyps had fundic gland polyp and $36.36 \%$ had hyperplastic polyp). All hyperplastic polyps were seen in antrum of stomach. One patient had both fundic gland polyp and hyperplastic polyp in the same time.
Duodenal adenoma was seen in $25 \%$ (7 patients). Tubular adenoma with low grade dysplasia was seen in $57.13 \%$ of patients with duodenal adenomas and tubulovillous adenoma with low grade dysplasia was seen in $42.8 \%$ of patients with duodenal adenomas.

Adenoma of papilla (peri-ampullary) was seen in one 22 year old male patient who was in stage one of spigelman stage classification. There was no patient with duodenal or gastric cancer, in our study. Fundic gland polyps were the most common polyps. Histology of polyps and location of lesions were demonstrated in table 2 .

According to the Spigelman classification, 6 patients were in stage 1 (with 4 points, $85.7 \%$ ), one patient was in stage 2 (with 5 points, 14.2\%) from 7 patients with duodenal Adenoma.

11 patients had non polyp in upper GI endoscopy $(39.28 \%)$. In contrast 1 patient had both gastric and duodenal polyp (3.56\%).

The most common site of polyps in duodenum was 2 th part of duodenum (7 patients). No patient with normal appearance of papilla had periampullary Adenoma. All the gastroduodenal polyps had sessile appearance.

One 36 year old female patient had extra colonic manifestation (Osteoid stoma in frontal bone and desmoids tumor in left ureter, Gardner syndrome).

Among 105 gastric polyps, the sizes of 3 polyps (2.9\%) were between $5-10 \mathrm{~mm}$ and the remainders were smaller than $5 \mathrm{~mm}(97.1 \%)$. Also in one patient the size

Table 1. Age at Diagnosis of FAP

\begin{tabular}{lcc}
\hline Age & Frequency & Percent \\
\hline $15-20$ & 9 & $32.10 \%$ \\
$20-25$ & 5 & $17.90 \%$ \\
$25-30$ & 3 & $10.70 \%$ \\
$30-40$ & 1 & $3.60 \%$ \\
$>40$ & 10 & $35.70 \%$ \\
Total & 28 & $100 \%$ \\
\hline
\end{tabular}

Table 2. Characteristics of Gastro Duodenal Polyps in FAP Patients

Number of patients (\%)

\begin{tabular}{ll}
\hline Gastric Polyp & \\
*Fundic gland polyp & $8(28.57 \%)$ \\
*Hyperplastic polyp & $4(14.4 \%)$ \\
*Tubular Adenoma(low grade) & $0(0 \%)$ \\
*Tubular Adenoma (High grade) & $0(0 \%)$ \\
Duodenal Polyp & \\
**Tubular Adenoma(low grade) & $4(14.28 \%)$ \\
**Tubular Adenoma (High grade) & $0(0 \%)$ \\
**Tubulovillous Adenoma(low grade) & $3(10.71 \%)$ \\
**Tubulovillous Adenoma(high grade) & $0(0 \%)$ \\
**Villous Adenoma & $0(0 \%)$ \\
Site of polyp & $2(7.1 \%)$ \\
Cardia of stomach & $7(25 \%)$ \\
Fundus of stomach & $4(14.3 \%)$ \\
Body of stomach & $5(17.9 \%)$ \\
Antrum of stomach & $1(3.6 \%)$ \\
Bulb of duodenum & $7(25 \%)$ \\
${ }^{2}$ th part of duodenum & $2(7.1 \%)$ \\
${ }^{3}$ th part of duodenum & $1(3.6 \%)$ \\
Ampulla of vater (peri Ampullary) & \\
\hline
\end{tabular}


of duodenal polyps was between $5-10 \mathrm{~mm}$ and the rest were smaller than $5 \mathrm{~mm}$.

\section{Discussion}

According to the results of present study, gastric polyps were distinguished about $40 \%$ of FAP patients and duodenal adenoma was seen in $25 \%$ of them. In the researches done till now the prevalence of gastroduodenal polyps in FAP patients has been reported higher than general population. Also the risk of progression of the mentioned polyps to the cancer (especially duodenal polyps and polyps of Ampulla of vater) has been considered higher, compared with polyps of stomach and duodenum in general population. In this study the prevalence of gastroduodenal polyps in Iranian patients affected by FAP has been evaluated. Fundic gland polyps are the most common polyps in FAP patients. The prevalence of Fundic gland polyps is about $50 \%$ (Park et al., 2011). But these polyps have been considered to have low potential for malignant changes (Kashiwagi and Spigelman., 2000). However, in one study $41 \%$ of FAP patients with fundic gland polyps had dysplastic changes (38\% low grade dysplasia and3\% high grade dysplasia) (Bianchi et al., 2008).

In a Korean study, the prevalence of FGPs in FAP patients was reported $25.7 \%$. In our study the prevalence of fundic gland polyps was $28.57 \%$ and no dysplasia or malignancy was seen in the pathology study of these polyps. The prevalence of gastric Adenoma has been reported 6-14\% in FAP patients (Park et al., 2011). Gastric Adenomas are usually located in the antrum of stomach and may be precursor of malignancy (Kashiwagi and Spigelman., 2000).

In this present study there were 4 patients $(14.2 \%)$ with hyperplastic polyp of stomach and all of hyperplastic polyps were in antrum of stomach. The prevalence of Gastric cancer in FAP patients in previous Asian study was higher than general Asian population (4.2\% in Korea, $2.1 \%$ in Japan) (Park et al., 2011). In our studies there was no patient with gastric cancer.

According to the western reports, duodenal adenomas can be found in $30-70 \%$ of FAP patients and the lifetime risk of these lesions approaches $100 \%$ (Park et al., 2011). The incidence of duodenal cancer is about 0.01 $0.04 \%$ in general population (Sexe et al., 1996) and FAP patients have 100-330 fold higher risk of duodenal cancer compared with the general population (Offerhaus et al., 1992).

A study showed that $65 \%$ of patients had duodenal adenoma (mean age $38 \mathrm{y}$ ) at the first endoscopy and two patients had asymptomatic duodenal cancer (Bulow et al., 2004). also another prospective study reported that $90 \%$ or more of patients were affected with duodenal polyposis and $10 \%$ had stage 4 polyposis, while $20 \%$ had stage 1 and the remainder had stage 2 or 3 (Kashiwagi and Spigelman., 2000). In numerous studies the progression of spigelman stage with time in FAP patients has been proved (Bulow et al., 2004; Kashiwagi and Spigelman., 2000).

In our study the prevalence of duodenal polyps was 25 $\%$ (7 patients), which it was higher than Korean patients but lower than western reports.

$85.7 \%$ of FAP patients (mean age: $29 \mathrm{y}$ ) with duodenal polyps had stage 1 of spigelman classification in our study. One patient had a polyp (tubular Adenoma) in papilla of duodenum. And no patient had duodenal cancer or duodenal adenoma with high grade dysplasia. Desmoids tumors occur at a rate of $10-15 \%$ in patients with FAP (Brueckl., 2005). In our research $3.57 \%$ of patients had desmoids tumor (one patient). In this study $67.85 \%$ of patients had gastric or duodenal polyps. All of duodenal polyps had low grade dysplasia.

Our findings are compatible with the findings of other researches that show that the prevalence of gastroduodenal polyps is high in FAP patients.

In conclusion, findings of this study indicated that the prevalence of gastroduodenal polyps in Iranian FAP patients is high and dysplasia has been seen in duodenal polyps of these patients. Therefore, it seems that routine gastroduodenal endoscopy in FAP patients would be necessary. Further studies with long time consecutive follow-up endoscopies would be useful for evaluation of the risk of progression of polyps to cancer and increase the number and size of polyps.

\section{Acknowledgements}

We show our gratitude to Taleghani Hospital for providing the data.

\section{References}

Bianchi LK, Burke CA, Bennett AE, et al (2008). Fundic gland polyp dysplasia is common in familial adenomatous polyposis. Clin Gastroenterol Hepatol, 6, 180-5.

Brueckl WM, Ballhausen WG, Fortsch T, et al (2005). Genetic testing for germline mutations of the APC gene in patients with apparently sporadic desmoid tumors but a family history of colorectal carcinoma. Dis Colon Rectum, 48, 1275-81.

Bulow S, Bjork J, Christensen IJ, et al (2004). Duodenal adenomatosis in familial adenomatous polyposis. Gut, $\mathbf{5 3}$, 381-6.

Cordero-Fernandez C, Garzon-Benavides M, Pizarro-Moreno A, et al (2009). Gastroduodenal involvement in patients with familial adenomatous polyposis. Prospective study of the nature and evolution of polyps: evaluation of the treatment and surveillance methods applied. Eur J Gastroenterol Hepatol, 21, 1161-7.

Church JM, McGannon E, Hull-Boiner S, et al (1992). Gastroduodenal polyps in patients with familial adenomatous polyposis. Dis Colon Rectum, 35, 1170-3.

Garrean S, Hering J, Saied A, et al (2008). Gastric adenocarcinoma arising from fundic gland polyps in a patient with familial adenomatous polyposis syndrome. Am Surg, 74, 79-83.

Goedde TA, Rodriguez-Bigas MA, Herrera L, Petrelli NJ (1992). Gastroduodenal polyps in familial adenomatous polyposis. Surg Oncol, 1, 357-61.

Jung SH, Chung WC, Kim EJ, et al (2010). Evaluation of nonampullary duodenal polyps: comparison of non-neoplastic and neoplastic lesions. World J Gastroenterol, 16, 5474-80.

Kashiwagi H, Spigelman AD (2000). Gastroduodenal lesions in familial adenomatous polyposis. Surg Today, 30, 675-82.

Nizam ZM, Abdul Aziz AA, Kaur G, et al (2013). Contribution of the MLH1 -93G>a promoter polymorphism in modulating susceptibility risk in malaysian colorectal cancer patients. 
Seyed Reza Fatemi et al

Asian Pac J Cancer Prev, 14, 619-24.

Offerhaus GJ, Entius MM, Giardiello FM (1999). Upper gastrointestinal polyps in familial adenomatous polyposis. Hepatogastroenterology, 46, 667-9.

Offerhaus GJ, Giardiello FM, Krush AJ, et al (1992). The risk of upper gastrointestinal cancer in familial adenomatous polyposis. Gastroenterology, 102, 1980-2.

Park SY, Ryu JK, Park JH, et al (2011). Prevalence of gastric and duodenal polyps and risk factors for duodenal neoplasm in korean patients with familial adenomatous polyposis. Gut Liver, 5, 46-51.

Poovorawan K, Suksawatamnuay S, Sahakitrungruang C, et al (2012). Colon cancer prevention by detection of APC gene mutation in a family with attenuated familial adenomatous polyposis. Asian Pac J Cancer Prev, 13, 5101-4.

Pourhoseingholi MA, Faghihzadeh S, Hajizadeh E, et al (2009). Bayesian estimation of colorectal cancer mortality in the presence of misclassification in Iran. Asian Pac J Cancer Prev, 10, 691-4.

Samalavicius NE (2008). Extracolonic Manifestations in Familial Adenomatous Polyposis. Govaresh, 8, 178-83.

Sexe RB, Wade TP, Virgo KS, Johnson FE (1996). Incidence and treatment of periampullary duodenal cancer in the U.S. veteran patient population. Cancer, 77, 251-4.

Somi MH, Golzari M, Farhang S, et al (2014). Gastrointestinal cancer incidence in East Azerbaijan, iran: update on 5 year incidence and trends. Asian Pac J Cancer Prev, 15, 3945-9.

Spigelman AD, Talbot IC, Penna C, et al (1994). Evidence for adenoma-carcinoma sequence in the duodenum of patients with familial adenomatous polyposis. The Leeds Castle Polyposis Group (Upper Gastrointestinal Committee). $J$ Clin Pathol, 47, 709-10. 